Voix et Images

voixetimages

\title{
Cher Survenant... : Germaine Guèvremont-Alfred DesRochers (1942-1951)
}

Yvan G. Lepage

Volume 16, numéro 1 (46), automne 1990

Les correspondants littéraires d'Alfred DesRochers

URI : https://id.erudit.org/iderudit/200874ar

DOI : https://doi.org/10.7202/200874ar

Aller au sommaire du numéro

Éditeur(s)

Université du Québec à Montréal

ISSN

0318-9201 (imprimé)

1705-933X (numérique)

Découvrir la revue

Citer cet article

Lepage, Y. G. (1990). Cher Survenant... : Germaine Guèvremont-Alfred DesRochers (1942-1951). Voix et Images, 16(1), 64-78.

https://doi.org/10.7202/200874ar d'utilisation que vous pouvez consulter en ligne.

https://apropos.erudit.org/fr/usagers/politique-dutilisation/ 


\section{Cher Survenant... : Germaine Guèvremont-Alfred DesRochers (1942-1951)}

\section{par Yvan G. Lepage, Université d'Ottawa}

C'est vers 1938, sans doute grâce à son poste de chef du secrétariat de la Société des écrivains canadiens, que Germaine Guèvremont paraît avoir fait la connaissance d'Alfred DesRochers ${ }^{1}$. Leurs routes ont dû se cróiser à Montréal, lors d'une assemblée générale de la Société, Alfred DesRochers étant le délégué de la branche estrienne de cette association nationale.

Née le 16 avril 1893, Germaine Guèvremont avait huit ans et demi de plus que DesRochers, mais, si l'on excepte les textes qu'elle avait fait paraitre entre 1926 et 1935 , alors qu'elle était correspondante au Courrier de, Sorel, on peut dire qu'elle n'avait encore rien publié qui pût lui assurer une quelconque audience dans le monde des lettres. En revanche, DesRochers s'était déjà fait un nom et une réputation, d'abord comme poète, grâce à ses deux recueils, l'Offrande aux vierges folles (1928) et À l'ombre de l'Orford (1929), puis comme critique et théoricien de la littérature, ainsi que le révèlent son abondante correspondance et les nombreux articles qu'il publia dans les périodiques, à commencer par la Tribune de Sherbrooke, journal auquel il collabora, sauf quelques interruptions, de 1925 à 1950.

À l'instar de nombreux écrivains québécois des années trente et quarante, Germaine Guèvremont trouva en DesRochers un véritable maître à penser, un mentor sur lequel elle put compter totalement, au point qu'elle n'eût rien publié sans son accord préalable. Il n'est, pour s'en rendre compte, que de parcourir les 111 lettres et billets

1 Cette hypothèse est en un sens corroborée par la remarque suivante, qu'on lit dans une lettre du 21 avril 1946, jour de Pâques: Une séance de lecture, comme nous en avions il y a une dizaine d'années, ne serait pas de trop. C'est si long à expliquer par lettre. Il y a tant de situations qui demandent de la sympathie. Mais si c'est bien vers 1938 (année où commencent à paraître les contes qui formeront le recueil En pleine terre) que Germaine Guevremont et DesRochers se rencontrèrent, sans doute par l'entremise de Françoise Gaudet-Smet et de la revue Paysana, on peut s'étonner de ne trouver aucune lettre antérieuse à septembre 1942. 
qu'elle lui a adressés, entre le 16 septembre 1942 et le 15 janvier 1951, et qui se trouvent aujourd'hui dans le fonds Alfred DesRochers, aux Archives nationales du Québec à Sherbrooke. Cette période s'étend approximativement de la parution d'En pleine terre (13 août 1942) au début du séjour de DesRochers chez Françoise Gaudet-Smet, à Claire-Vallée (1952). Après ce séjour, DesRochers s'installa définitivement à Montréal, ce qui mit fin à la correspondance que Germaine Guèvremont entretint avec lui ${ }^{2}$.

Ces lettres, de longueur variable, allant de l'épître à la simple carte de Noël (décembre 1948), sont quelquefois autographes (24), mais le plus souvent dactylographiées (88). Dans ce dernier cas, Germaine Guèvremont utilise tantôt sa propre machine à écrire, tantôt celle du secrétariat de la Société des écrivains canadiens (3425, rue Saint-Denis, d'abord; puis 535, avenue Viger, à partir de 1944). Tous les formats et tous les types de papier servent, du très petit $(9 \times 13 \mathrm{~cm})$ au très grand $(21 \times 33 \mathrm{~cm})$, de la banale feuille blanche au papier à lettres aux armes des Chevaliers de Colomb - (dont son mari semble avoir été membre) ou à en-tête de la revue Paysana (à laquelle elle collabora beaucoup). Mais l'épistolière paraît avoir eu un faible pour un type particulier de papier, d'un blanc tirant sur le gris, aux dimensions modestes $(10,5 \times 15,5 \mathrm{~cm})$, tout à fait approprié à l'expédition de billets ou de courtes lettres rédigées en toute hâte (17 sur 112).

Peu de lettres portent une date; nous avons dû nous livrer à un long et patient examen pour tenter de découvrir à quel moment chacune avait été rédigée. Nous y sommes généralement parvenu, mais les dates proposées restent évidemment hypothétiques (d'où l'addition du point d'interrogation entre crochets). Onze lettres et billets sont toutefois impossibles à dater dans l'état actuel de nos connaissances, ne serait-ce que parce que certains ne contiennent aucun indice.

La plupart des lettres que Germaine Guèvremont adressa à DesRochers (73 précisément) s'échelonnent sur une période de trois ans, de janvier 1944 à décembre 1946. De ce fait, elles concernent essentiellement le Survenant ${ }^{3}$ et Marie-Didace ${ }^{4}$ et elles constituent une mine de renseignements sur la gestation de ce diptyque romanesque, une mine d'autant plus précieuse qu'elle est toujours inédite.

2 Une cent-douzième et dernière lettre de Germaine Guèvremont à DesRochers est datée du 19 février 1960; elle a été écrite à Saint-Sauveur-des-Monts.

3 Germaine Guevremont, le Survenant, édition critique par. Yvan G. Lepage, Montréal, Presses de l'Université de Montréal, 1989, 367 p. (Bibliotheque du Nouveau Monde). Toutes nos références renvoient à cette édition, désignée par le sigle $\mathbf{S}$ et suivie de la mention de la page.

4 Germaine Guevremont, Marie-Didace, Montréal, Fides, 1980, 229 p. (Bibliothèque québécoise). Toutes nos références renvoient à cette édition, désignée par le sigle MD et suivie de la mention de la page. 
En DesRochers, Germaine Guèvremont admirait l'homme fait de contrastes: poète raffiné et cultivé, mais aussi shantyman; guide littéraire, mais également fils déchu; journaliste et coureur de bois. Ce nomade ne dédaignait pas les produits de l'orge et du houblon, où il puisait son inspiration. S'il existe quelque part un modèle du Survenant, c'est d'abord chez DesRochers qu'il faut le chercher. Symbole et double du Grand-dieu-des-routes, il en fut aussi l'inspirateur, le père spirituel, celui sans qui Germaine Guèvremont n'aurait pu donner la vie. Quand on découvre, par ailleurs, que la plupart des lettres qu'elle lui adresse - et qu'elle signe Marie-Amanda portent comme appel, tantôt Cher Survenant, tantôt Cher père Didace, on mesure mieux l'ambiguïté du personnage, à la fois modèle et père.

Nous avons déjà eu l'occasion, dans l'introduction de notre 'édition, d'examiner les lettres qui ont trait à l'élaboration du Survenant (janvier 1944-7 avril 1945); nous n'y reviendrons pas ici. C'est plutôt la suite de cette correspondance qui nous intéressera cette fois. Bien qu'il soit amputé d'une voix - celle de DesRochers, dont les lettres à Germaine Guèvremont ne semblent pas avoir été conservées ${ }^{5}-$, le "dialogue" que constitue cette correspondance des années 1945 et 1946 éclaire d'un jour tout à fait neuf la composition de Marie-Didace, tout au moins certains chapitres.

Déjà, en 1944, au moment où elle se livrait à la rédaction du Survenant, Germaine Guèvremont écrivait parallèlement quelques épisodes qui allaient prendre place dans Marie-Didace:

Mon cahier est bourré de notes. [...] Ainsi quand le Survenant, - (pour vous donner une idée de la dernière note enregistrée) quand le Survenant bat un Provençal, les femmes demandent au père Didace d'aller l'arrêter. Mais le père Didace trouve que son Survenant, c'est un "maudit bon homme». Il s'approche de lui et lui dit:

"Bats-le. Fesse à ta force. Aie pas peur. S'il te fait arrêter, je cautionnerai pour toi». [S, p: 187]

Quand Angélina trouvera le bout de gazette avec le portrait de Venant, et sa décoration "mort au champ d'honneur", elle reprendra ce que disait son Grand-dieu-des-routes: cherchezmoé [pas] dans les fossés, vous me trouverez pas. Je mourrai etc. etc. [MD, p. 199; S, p. 265]

5 On n'en trouve aucune dans le fonds Alfred DesRochers, ANQ-S, si ce n'est une copie dactylographiée de la lettre fictive qu'il fit paraitre dans la revue Paysana (vol. V, no 12 , février 1943, p. 13), et qui s'intitule "Lettre d'un Ouistipouf". Elle est adressée à sa "Chère Amanda " et elle emprunte le ton passionné d'une jeune recrue souffrant d'être séparée de sa fiancée à l'occasion de la Saint-Valentin. 
Je lis la mort de Didace à ma belle-mère [MD, p. $164 \mathrm{s.}]^{6}$. Elle pleure et dit: Si je peux avoir le bonheur de lire ce livre-là [...]. (juin 1944 [?])

Le Survenant parut le 7 avril 1945. Dès juin, la correspondance de Germaine Guèvremont fourmille d'allusions à Marie-Didace et aux pages qu'elle soumet à l'appréciation de DesRochers, son cher Didace:

[:..] ne soyez pas surpris si vous recevez demain ou aprèsdemain [...] quelques pages. La crise est finie. Mais je vous demande en grâce de toujours tenir compte qu'il ne s'agit là que de pages toutes nues. Il faudra les habiller avant qu'elles. soient montrables.

Merci de votre patience et jattends de vos nouvelles, un de ces jours prochains. (juin 1945 [?])

Et elle signe: Sincèrement, Marie-Amanda. Dans une autre lettre de lá même époque, elle écrit:

pour vous donner une "prévue» des chapitres à venir: dans la querelle d'Amable avec le père Didace, celui-ci s'arrêta saisi:

La joie rajeunit sa voix: (Didace à Amable) Tu partirais, toi? Mais aussitôt allumée, aussitôt éteinte, la joie retomba:

- Tu sais pas seulement tenir un outil comme il faut. Et quand tu le laisses tomber, c'est toujours le manche qui est en faute, jamais ta main. [MD, p. 96]

Et pour faire écho à cette scène, elle ajoute, vers le 25 juin: $M e$ permettrez-vous de vous dire un mot de l'Acayenne; si cela ne vous ennuie pas, après la chicane entre Didace et Amable: - D'abord que c'est de même, je vas vous régaler de mon absence. [MD, p. 96]

D'autres lettres de juin-juillet 1945 font référence au départ d'Amable et aux chapitres qui suivent, comme, par exemple, celle du 27 juillet:

[...] la présente est donc pour vous envoyer un beau livre [Jean Ménadieu de Gontran de Poncins], en attendant Marie-Didace qui se porte bien, ainsi que toute la famille. Amable-Didace est parti du chenal. Angélina n'a pas de consolation en parole à donner à Alphonsine. Seulement, un soir, elle lui apporte une plante en pot, pensant que ça la distrairait, l'occuperait:

- Je t'ai apporté un petit bouquet. Prends en soin parce que si.je m'aperçois que tu le laisses dépérir, je viendrai le chercher, je te le promets. [MD, p. 112]

6 Un extrait de ce chapitre parut dès juin 1945: «Une dernière passée», Gants du ciel, $n^{\circ} 8$, juin 1945 , p. 41-46. 
Comme vous voyez je saute d'une île à l'autre et je ne puis vraiment vous envoyer [que] ce que vous appelleriez des taches de peinture, mais j'ai "l'état de grâce du romancier".

On comprendra mieux cette allusion aux taches de peinture quand on aura rappelé que Valentine Labelle, mère de Germaine Guèvremont, s'adonnait à la peinture et que la romancière a maintes fois déclaré qu'elle composait un livre à la manière de l'artiste peignant sa toile:

[...] pour ma part je pourrais dire que j’emploie le procédé d'un artiste-peintre... je veux dire, que ce dernier ne commence pas toujours son tableau dans un coin spécifique de la toile, mais n'importe où, là où sa fantaisie le pousse ou son inspiration le commande... au beau milieu, au bas, n'importe où sur la toile. C'est ainsi que j'écris constamment des paragraphes, même des chapitres entiers, détachés, et qui plus tard trouvent place dans la trame de mon roman. ${ }^{7}$

Ce mode d'écriture convient certes mieux au conte et à la nouvelle qu'au roman, lequel requiert du souffle. C'est pourtant ce procédé; proche du pointillisme, que Germaine Guèvremont a appliqué, aussi bien au Survenant qu'à Marie-Didace, ainsi que nous le révèle sa correspondance. Si le Survenant n'en a pas trop souffert, en raison de son intensité et de la fascination qu'exerce le Grand-dieu-des routes, on ne saurait en dire autant de Marie-Didace, œuvre moins cohérente et moins harmonieuse. Ces défauts, il ne faut toutefois pas les exagérer, car le rythme, beaucoup plus rapide que celui du Survenant ${ }^{8}$ (comme elle le déclare elle-même dans sa lettre du 27 juillet 1945), vient les atténuer.

Après les chapitres 11 et 13 de la première partie de MarieDidace, ce sont tour à tour les chapitres 3 et 1 que Germaine Guèvremont semble rédiger, à en juger par ses lettres d'août et de septembre 1945. Mais c'est le chapitre premier, le commencement, qui paraît lui causer le plus de soucis: elle y revient à plusieurs reprises, exposant ses craintes et ses espoirs:

Voici Marie-Didace... avec mes craintes et mes espoirs. Et même si je voudrais [sic] vous dire le contraire, je vous demande de ne ménager ni les uns, ni les autres. Mon mari

7 Rosaire Dion-Lévesque, "Quinze minutes avec Germaine Guèvremont", le Travailleur, 27 juillet 1950, p. 1. Voir aussi André Langevin, "Madame Germaine Guèvremont ", Notre temps, 12 juillet 1947, p. 3, et Louis Pclletier-Dlamini, "Germaine Gudvremont. Rencontre avec l'auteur du Survenant ", Châtelaine, vol. VIII, $n^{\circ} 4$, avril 1967 , p. 84.

8 . Deux lettres de Germaine Guèvremont, d'avril 1944, laissent entendre que DesRochers trouvait le début du Survenant trop lent, reproche qu'il formula de nouveau dans le compte rendu qu'il donna de ce premier roman dans la Revue populaire, vol. XXXVIII, $n^{\circ} 6$, juin 1945, p. 58. 
l'aime. J'attends votre jugement. Puisque c'est le point de départ de toute une envolée. La crainte de Phonsine s'y dessine. Elle ne fera que s'accentuer. (août 1945 [?]) ${ }^{9}$

Si l'exaltation de la vie caractérise le Survenant, les forces vives de l'amour et de la fécondité cèdent la place à la mort dans MarieDidace. La flamboyante figure d'Éros ${ }^{10}$ une fois évanouie, Thanatos entre en scène, poussant devant lui les figures grimaçantes de la crainte, de l'angoisse et de la folie, toutes œuvres souterraines de la Mort. Car si la joie est bondissement, la-névrose se confond avec la descente, ainsi que le montre la chùte dans le puits qui hante le sommeil de Phonsine. Et c'est précisément l'inexorable marche des puissances chtoniennes que Germaine Guèvremont a choisie comme fil conducteur de Marie-Didace, cette œuvre où domine le personnage de Phonsine, au point que la romancière songea un temps à en changer le titre, substituant "Alphonsine" à "Marie-Didace", la mère folle à la fille orpheline: autant dire la mort à la vie.

Le malheur, Phonsine le comprend aussitôt, c'est avec l'Acayenne qu'il pénètre sous le toit des Beauchemin:

Pour en revenir à mon sujet, dans mon village, et le vôtre aussi probablement, il n'y avait que deux heures auxquelles un veuf pouvait convoler en secondes ou troisièmes noces: le soir, ce qui était une sorte dé cachette, et le matin, à cinq heures. J'avais songé à ce que Phonsine, entendant remuer dans la chambre voisine, quand elle ne dort pas, remarquât ou passât la remarque ou à peu près: "Ton père qui se lève déjà !» Amable: "Il doit s'en aller à la passe». Phonsine: "Ya$t$-il du danger qu'il parte, un matin, de même, pour se remarier?" Et ainsi de suite... Je croyais que de pénétrer au plus tôt dans l'histoire (Get into your story as soon as possible) était une bonne chose. Il est vrai que l'éclaircissement au sujet du mariage $d u$ père Didace ne changerait rien. ( $1^{\text {er }}$ et 2 septembre 1945)

DesRochers dut trouver cette remarque pertinente puisque Germaine Guèvremont renonça à développer la scène esquissée ici, pour ne conserver que la réplique suivante: Remarque bien ma parole, lui dit Phonsine. À la première nouvelle, il nous ressourdra marié. Et quand le couple pénètre dans la cuisine, au début du deuxième chapitre, c'est pour tomber en arrêt devant une étrangère qui déclare en souriant: Je suis la femme au père Didace. Le lecteur a compris (en

9 Cf. Marie-Didace, $1^{\mathrm{re}}$ partie, chapitre 1: Les bras en couronne sur la tête, Phonsine ne dormait pas. Inquiète, elle epiait dans la nuit les moindres bruits de la maison [...].

10 Voir Robert Major, “Le Survenant et la figure d'Éros dans l'œuvre de Germaine Guèvremont ", Voix \& images, vol. II, $n^{\circ} 2$, décembre 1976, p. 195-208. 
même temps qu'Amable et Phonsine): le père Didace a convolé en secondes noces à cinq heures du matin, dans le plus grand secret. Germaine Guèvremont put alors écrire, quelque temps après:

Je suis si avancée dans mon histoire qu'il me prend des rages de ne pouvoir le crier. J'ai tassé le commencement. J'en ai ôté tout ce qu'il y avait de tatillon, d'Alphonsinard dans les coins ronds à tel point que je pense que vous serez content de moi [...]

En tout cas, ça va bien, gardez-moi toute votre patience, jusqu'au bout. Et votre encouragement.

À bientôt. Si vous ne venez pas dans ce bout-ci, il se peut, si ça concorde avec vos plans, que je pousse (le 28) une pointe dans vos alentours. On pourrait mettre l'O.K. à bien des pages. (automne 1945 [?])

Les quelques lettres de l'automne 1945 concernent moins MarieDidace que les projets de réédition d'À l'ombre de l'Orford à la Société des éditions Pascal, fondée et dirigée par Gérard Dagenais. Ces projets échouèrent et le recueil fut finalement soumis à Fides, qui le publia trois ans plus tard, en 1948, dans la collection du Nénuphar.

À partir de décembre 1945 , et tout au long de l'année 1946 , Marie-Didace occupe une place centrale dans la majorité des lettres de Germaine Guèvremont. C'est ainsi qu'elle évoque pêle-mêle les chapitre 12 et 6 de la première partie et 4 et 3 de la deuxième partie, dans une lettre écrite après le 9 décembre 1945, où elle explique à son: cher père Didace les raisons qui ont retardé l'écriture de Phonsine:

À l'heure où je commençais à désespérer de ne plus voir votre écriture, pas même votre croix au bas d'un papier, je trouve une belle lettre frétillante de vie, avec un gros 9 [9 décembre] qu'on dirait bien engraissé pour lè fêtes [...].

Si vous voulez être franc au sujet des raisons qui ont retardé l'écriture de Phonsine, ajoutez donc des mois de désespoir muet devant des critiques comme celles de la Presse ${ }^{11}, d u$ Canada ${ }^{12}$ et de la Revue dominicaine ${ }^{13}$. Maintenant je connais un beau rêve, mais aussi la peur folle de toutes mes extases; de ne pas me rendre jusqu'au bout, de n'en pas

11 Léon Franque [pseudonyme de Roger Champoux], "Le Survenant ", la Presse, 12 mai 1945 , p. 30.

12 Berthelot Brunet, “L'exotisme de ma paroisse », le Canada, 21 mai 1945, p. 4.

13 Albert Saint-Pierre, "L'esprit des livres. Germaine Guèvemont, le Survenant. Roman régionaliste", Revue dominicaine, vol. LI, n² 2, septembre 1945, p. 121 122. 
connaître la fin. Ainsi quand Amable part pour Sorel, Phonsine le regarde partir. Amable s'accroche et tombe les deux genoux dans la neige. (Il ne doit plus jamais revenir au Chenal du Moine). Phonsine se cache pour que son mari ne la voie pas parce qu'elle a peur qu'il revienne sur ses pas. Maintenant qu'il est parti, que le coup est donné, qu'il s'en aille! Mais quand elle ne le voit plus sur la route, elle regarde les deux trous dans la neige et ne peut en détacher ses yeux. C'est comme si toute la faiblesse de l'homme y fût enfouie. [MD, p. 104-105]

Dès le début du livre, un peddleur arrive à la maison. Moitié juif, moitié syrien, et le reste canayen. Ah! il y aurait trop à écrire là-dessus. Que je vous dise seulement qu'Alphonsine qui avait envie de tout, à la fin ne prend qu'un peigne de corne rose (rond) et un coupon de flanelette, tandis que l'Acayenne qui ne voulait rien hérite du beau coupon d'alpaca brun. [MD, p. $43 \mathrm{~s}$.]

[...] À la fin du livre, Phonsine croit qu'elle a tué l'Acayenne, mais celle-ci a pris plus que la dose de pilules prescrite. [MD, p. $188 \mathrm{~s}$.

[...] Pour finir, quand le père Didace est sur le point de mourir, il parle à Phonsine et lui dit: "Fais-toi aimer de ta petite. C'est pas tout de nourrir un enfant, de l'élever, faut se faire aimer...» [MD, p. 175]

Les quatres scènes esquissées ici se retrouvent effectivement dans la version finale de Marie-Didace. Il en est une cinquième, en revanche, qui ne sera pas retenue, mais que, dans la même lettre, Germaine Guèvremont évoque en ces termes:

[...] Et puis, ce n'est pas tout, la grande nouvelle c'est que le Survenant va revenir. Un dimanche, tandis que tout le monde du Chenal est à la messe, il a guetté l'arrivée des gens à l'église et s'est rendu au Chenal jusqu'à la maison de Didace. Il rencontre Marie-Didace qui ne le connaît pas; il lui parle; lui demande son nom, puis "Va dire à ton grand'père que quelqu'un le fait demander au chemin." "Mon grand'père est mort", répond Marie-Didace. Et ton père, où est-il, il n'est pas à la messe? Alors il s'informe de tout le monde du Chenal, d'Angélina, si elle est mariée... Et il s'en retourne sans avoir revu personne. Marie-Didace dira à sa mère qu'un soldat lui a parlé, mais Phonsine ne fait que la gronder d'avoir parlé à un étranger, sans chercher à savoir qui l'homme était... etc., etc. Qu'en pensez-vous? Alors quand Angélina découvrira le portrait du Survenant mort au champ d'honneur, le lecteur comprendra, sans même savoir son nom. [MD, p. 199 s.]

Rien ne révèle mieux que cette page la difficulté, pour la romancière, d'admettre le départ définitif de son personnage d'élection. Obsédée par 
le Survenant, elle aura éprouvé l'incoercible désir de le faire apparaître une toute dernière fois avant de se résigner à le faire mourir au champ d'honneur. Et dans une lettre subséquente, de décembre 1945 ou de janvier 1946, elle s'écrie avec enthousiasme:

Le Survenant est revenu... le miracle s'est accompli. Je ne vous envoie qu'une faible partie des pages, parce que tout simplement le temps me manque pour vous en copier davantage. Si vous trouvez que l'esprit y est, dites-moi le donc d'un seul mot qui me ferait bien plaisir.

Mais DesRochers dut finalement la convaincre de renoncer à cette supercherie romanesque, dont on croit entendre l'écho dans ces mots:

Didace ne broncha pas. Longtemps il avait espéré, et craint à la fois, le retour du Survenant, parce qu'en revenant au Chenal du Moine, malgré la joie que le père Didace en eût éprouvée spontanément, le grand-dieu-des-routes aurait triché. [MD, p. 150]

Et Germaine Guèvremont supprima cette scène qui évoquait le retour du Survenant, non sans se réserver toutefois le droit de ressusciter un jour son personnage, ce qu'elle se permit quatorze années plus tard, dans la dernière tranche de l'adaptation télévisée qu'elle donna de son œuvre ${ }^{14}$.

Une autre lettre de la même époque nous apprend que Germaine Guèvremont avait prévu, entre la petite Marie-Didace et son grand-père, un court dialogue apte à accentuer un trait du caractère de ce dernier:

Didace est toujours Didace: pour le premier livre, il est sur la tempérance: quand un étranger lui offre un coup, il refuse, mais à chaque fois, il dit au Survenant: "Prends le mien, Survenant». [S, p. 215] Il n'est pas pour laisser perdre ça.

Dans le deuxième livre, Blanche V. donne une cenne à MarieDidace pour la quête. Quand le curé est sur le point de passer, Didace pousse la petite: "Donne-la pas. Garde-la pour t'acheter du nananne. Donne-la pas.» (hiver 1945-1946 [?])

Ce détail humoristique, qui devait sans doute prendre place quelque part dans le chapitre de la deuxième partie, fut finalement sacrifié.

En février 1946, la romancière évoque tour à tour la scène du "bee» de piquage chez les Beauchemin ${ }^{15}$ et le récit de la visite de Didace chez

14 Cf. Le Survenant, téléroman diffusé à Radio-Canada, du $1^{\text {er }}$ octobre 1959 au 23 juin 1960; hebdomadaire, 30 minutes; réalisation de Denys Gagnon. Ce téléroman succédait à trois autres du même auteur, intitulés respectivement le Survenant (30 novembre 1954 -9 juillet 1957), Au Chenal du Moine (17 octobre 1957 - 10 juillet 1958) et Marie-Didace (25 septembre 1958 - 25 juin 1959).

15 Cf. Marie-Didace, $1^{\text {re }}$ partie, chapitre 8. Lettre de Germaine Guèvremont à DesRochers, 20 février 1945 [?]. 
le notaire ${ }^{16}$. Ce récit, qui occupe le début du chapitre quatorze de la première partie, Germaine Guèvremont y revient dans sa lettre du 2 mars 1946, en même tenps qu'elle annonce le chapitre suivant:

Ainsi je vous enverrai ces jours-ci le chapitre du notaire. Ce que j'ai pu l'émonder, grands dieux. Vous vous en rendrez compte. Puis la naissance de la petite suivra. Enfin, ça va. Et je vous remercie de m'accompagner toujours dans cette rude montée.

Comme elle le précise ici, une fois rédigé dans ses grandes lignes, chacun des chapitres de Marie-Didace est soumis à un patient travail de révision, de corrections et de retouches:

À mesure que j'avance dans l'écriture, j'ai l'impression que le livre s'élargit, s'aère, même si je n'ai pas le temps de toujours l'élargir, l'aérer tout de suite sur le papier, et de poser la frange de la poésie et les ornements de détail, enfin la finition. (2 mars 1946)

Comme l'artiste peintre, la romancière sent bien qu'il lui faut parfois un peu accentuer ça et là, sans déparer le récit ${ }^{17}$ et, d'autres fois, nourri[r] le récit qui [lui] apparaît comme schématique par bouts (lettre postérieure au 3 mars 1946 [?]). Mais elle n'èn recherche pas moins l'avis de son fidèle guide, ne ratant jamais une occasion de le remercier pour ses encouragements et ses élogieuses remarques.

Le jour de Pâques 1946, c'est une longue lettre qu'elle rédige; elle annonce à son correspondant qu'elle vient d'accepter l'offre que lui faisait Plon, pour la seconde fois, de donner une édition française du Survenant: refuser une telle offre, il me semble que c'est comme refuser.le baiser de sa mère et si la France ne compte plus pour nous, qu'est-ce qui compte? Par ailleurs, Plon lui demande de redonner à [son] prochain livre son premier titre: MARIE-DIDACE ${ }^{18}$. Revenant ensuite à l'œuvre en chantier, Germaine Guèvremont reparle du chapitre treize, dont il avait été question une première fois le 27 juillet 1945 :

[...] après le départ d'Amable, Didace respecte la gêne de Phonsine devant sa grossesse. La deuxième journée, il l'a passée en canot à entailler des plènes. (Car on fait les sucres en canot, à l'eau haute, et c'est unique! à remarquer que dans les grands romans canadiens, il n'y a jamais eu de sucres). (remarque à corriger si

16 Marie-Didace, $1^{\text {re }}$ partie, chapitre 14, p. 120 . L'allusion à ce récit se lit dans une note manuscrite au bas du verso d'une copie dactylographiée de la lettre de la Librairie Plon à Germaine Guèvremont, 25 février 1946, lui proposant une édition française du Survenant dans la collection "l'Épi ».

17 Lettre de Germaine Guèvremont à DesRochers, février ou mars 1946 [?]: Ainsi, poursuit-elle, dès que je parle d'Angélina, jajoute au bon moment - la boiteuse et, comme nous décidions, par ce samedi après-midi, je rappelle la Pelerine, la cloche de Sainte-Anne de Sorel, etc.

18 Marie-Didace est effectivement le titre du livre " $\grave{A}$ paraître " qu'annonçait la première édition du Survenant (Montréal, Beauchemin, 1945, p. 4). 
erronée). Le soir Phonsine qui ne peut dormir entend des coups de marteau dans le fournil. Elle se lève et va regarder par la fenêtre. Son beau-père est en train de réparer le ber qu'il a descendu du grenier pour elle.. Mais il faudrait s'en parler, pour bien faire.

Les pages que je vous envoie, sans me satisfaire pleinement, vous, donnent une bonne idée du patron du chapitre. Il y a bien des coins à équarrir.

Ce qui me déroute c'est que vous ne semblez guère vous en tenir au barème que vous aviez établi verbalement:

ÉBLOUISSEMENT: téléphone

bon chapitre: lettre immédiate

nul: silence.

Mais les barèmes, ça doit être les descendants des promesses, de la même famille. (21 avril 1946)

Bien qu'intermittente, la collaboration de DesRochers à l'œuvre de Germaine Guèvremont tint bon, et la romancière continua à lui soumettre des "pages", si bien qu'à la fin de l'année 1946, elle en avait accumulé environ trois cents et qu'elle était en mesure de dresser un nouveau [?] plan du roman:

Pour en revenir à Marie-Didace, croyez-le ou non, je viens d'en faire un plan. La première partie comprend quatorze chapitres, et la deuxième, cinq. Comme j'ai à peu près 300 pages écrites, ça va. J'en rejeterai [sic] au moins 100. Et j'en ajouterai peut-être deux cents. Ce qui fait qu'il en restera... calculez s.v.p. ${ }^{19}$

19 Lettre de Germaine Guèvremont à DesRochers, novembre ou décembre 1946 [?]. Un canevas primitif de six pages dactylographiées est joint à la dactylographie de MarieDidace, conservée dans le fonds Alfred DesRochers, ANQ-S, sous la cote P0006, série 2, article 10. Coiffé du titre "Patron à l'œil de "Marie-Didace" ", ce canevas comporte 19 chapitres, avec l'indication "Deuxième partie» entre les chapitres neuf et dix. L'économie générale de ce "patron » differe profondément de celle du plan définitif, ainsi que le montre le tableau suivant (les chapitres de la première partie du texte final sont numérotés ici en chiffres arabes; ceux de la deuxième partie le seront en chiffres romains):

PATRON À L'EIL

Chap. 1

2

3

4

5 [Amable à Sorel 6 puis à Montréal]

7

8

9

Deuxieme partie

10
MARIE-DIDACE (texte final)

Chap. 1, 2

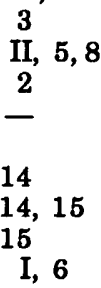

II 
Ce plan ne fut pas adopté tel quel; les transformations qu'il subit le firent passer de quatorze à quinze chapitres, pour la première partie, et de cinq à six pour la deuxième partie.

D'autre part, certaines pages furent effectivement rejetées, comme celles qui devaient développer le scénario suivant, tout plein du souvenir lancinant du Survenant:

Donc, dans le temps des fêtes, le Survenant revient pour tout le monde qui a un peu de cour, au Chenal du Moine, sans que son nom soit même prononcé, et plus présent que s'il eût été en chair et en os, car on ne le voit que sous son meilleur jour. À tout moment, Phonsine court à la fenêtre. Elle a toujours l'air de chercher. Après la messe de minuit, Marie-Amanda prend le fauteuil du Survenant et le met à part, dans un coin. À la soirée des Salvail, à l'heure des chansons, Bernadette à Odilon: Si je te paye un coup, vas-tu chanter? Odilon répond: Un coup? Pourquoi faire? Alors Bernadette lui ferme au nez la porte de la chambre des étrangers. Quand elle en sort, elle rit aux éclats mais ses yeux sont rougis et va trouver Angélina, assise seule en haut, près $d u$ tuyau de poêle. "Je me chauffe», dit-elle à B. qui lui demande pourquoi elle reste seule ainsi. Les deux veillent ensemble tandis qu'en bas, on danse et on chante. (novembre 1946 [?])

La même lettre contient par ailleurs deux autres scènes qui furent retenues. La première a trait au dialogue entre Didace et Angélina qu'on lit au chapitre neuf de la première partie, mais telle qu'elle se présente ici, cette esquisse differe beaucoup de ce qu'elle deviendra dans le roman:

Didace a fait deux ou trois voyages chez les Desmarais afin de parler seul à seule avec Angélina. Enfin, un bon jour, il la trouve assise auprès du poêle, à peigner ses longs cheveux. Dès qu'elle reconnaît le père Didace, elle ramène ses cheveux devant ses yeux et s'en fait comme un rideau pour qu'il ne voit [sic] pas son visage. À force de courage, elle finit par se composer un visage et relève ses cheveux. Le père Didace, malheureux, bourru et chagrin, finit par lui demander à brûle-pourpoint : "En effet, as-

$\begin{array}{ll}11 & \begin{array}{l}\text { [Alphonsine et Didace } \\ \text { à Montréal] }\end{array} \\ 12 & \text { III } \\ 13 & \begin{array}{l}\text { [Explication du testament] } \\ \text { et hallucination de Phonsine }\end{array} \\ 14 & \text { II } \\ 15 & \text { [Marie-Didace trouve la lettre] } \\ 16 & \text { VI } \\ 17 & \text { IV } \\ 18 \text { IU } \\ 19 \text { ["dernier chapitre, revu, } \\ \text { augmenté et corrigé] }\end{array}$


tu eu de ses nouvelles?" Au lendemain des rois, la vie du Chenal reprend son cours.

Quant à la seconde de ces scènes, son intérêt réside principalement dans le fait qu'elle nous permet de voir la romancière au travail:

Si je tiens absolument à aller au Chenal, c'est qu'on a ramassé les animaux sur la commune, mais qu'un seul cheval y reste, qui ne veut pas se laisser capturer. Il paraît qu'il est superbe, vu par les yeux de mon mari, mais je veux le voir par les miens. Valdombre dit que j'ai perdu beaucoup de temps à accorder tant de prix à chaque détail [...]. C'est son privilège. Mais c'est aussi le mien de prendre deux jours de mon temps et de mon argent pour aller voir un cheval farouche sur la commune.

Les hommes en causent, un jour. Odilon dit soudainement, en le regardant, la tête haute, la crinière au vent, qui défie les hommes: I me fait penser au Survenant!

Amable s'esclaffe mais le père Didace jette un Neveurmagne!, le seul du livre, PROBABLEMENT. Mais il pense: Lui l'aurait attrapé vite. C'est pas Amable...

Amable qui est le bon sens même ajoute

- Laissez-le donc faire. Il est pas' encore blessé: il a de quoi manger. Mais attendez que la neige prenne. Il va s'approcher du bord, ça sera pas long. [MD, p. 54]

On a souvent insisté sur le souci du détail qui caractérise l'art de Germaine Guèvremont et sur l'influence que Balzac exerça sur elle ${ }^{20}$ : cette page en constitue la meilleure des démonstrations, en même temps que s'y abolit la frontière qui sépare la fiction de la réalité.

L'année 1947 - qui verra la publication de Marie-Didace - s'ouvre sur l'attribution du prix Sully-Olivier de Serres pour l'édition française du Survenant et sur la décision de la direction des Éditions Fides de publier À l'ombre de l'Orford dans la collection du Nénuphar. Pour Germaine Guèvremont et pour Alfred DesRochers, ce sont de bonnes et heureuses nouvelles.

Jusqu'en juin, leurs rencontres durent se multiplier, ce qui expliquerait l'absence quasi totale de lettres au cours du premier semestre.

20 Voir, par exemple, Rita Leclerc, Germaine Guèvremont, Montréal, Fides, 1963, p. 81-82 (Écrivains canadiens d'aujourd'hui, 1); Pierre Girouard, Germaine Guèvremont et son cuvre cachée, Tracy, Cégep de Sorel-Tracy, 1984, p. 21-22, et Céline Légaré, "Confidences "filmées" d'une romancière. Germaine Guèvremont doit à Balzac l'art de situer ses personnages ", la Presse, 19 mars 1959, p. 18 [à propos de Germaine Guèvremont romancière, film de l'ONF réalisé par Pierre Patry et diffusé à Radio-Canada, le dimanche 22 mars 1959]. 
Le jeudi saint 3 avril 1947, Germaine Guèvremont envoie un mot à ses Chers amis DesRochers pour les informer de son arrivée à Sherbrooke: J'arriverai donc avec au-delà de 200 pages de textes. [...] Mais ne vous hâtez pas trop de sortir les grands mouchoirs de poche, ni de convoquer les braillardes, car plus ça va, moins je suis sûre de moi. Nous ne connaissons malheureusement pas les dernières étapes de l'élaboration du roman, mais on peut parier qu'elles donnèrent encore lieu à d'interminables discussions et à de nombreuses modifications de détail. Quoi qu'il en soit, la version dactylographiée conservée aux Archives nationales du Québec à Sherbrooke comporte 195 pages et un bon nombre de corrections d'auteur, mais aussi, au début surtout, quelques remarques et suggestions de la main même d'Alfred DesRochers. In s'agit d'une copie carbone du texte qui fut vraisemblablement remis à l'imprimeur en juillet [?] $1947^{21}$ et dont l'achevé d'imprimer est du 15 août.

Marie-Didace obtint un succès immédiat et total, personne n'osant plus exprimer la moindre réserve après la consécration française du premier volet du diptyque, ce qui fit dire à Germaine Guèvremont, sur un ton où perce un mélange de triomphe et d'amertume: Je reçois lettre sur lettre - Issalys ${ }^{22}$ aussi - de gens qui levaient le nez sur le Survenant. (lettre postérieure au 15 octobre 1947 [?])

Alors plurent les honneurs: médaille de l'Académie canadiennefrançaise (1947), élection à la même Académie (6 décembre 1948), publication de Marie-Didace à Paris (1949) et prix du Gouverneur général pour la traduction anglaise des deux romans (1950).

Cependant, après avoir porté et mis au monde Marie-Didace, Germaine Guèvremont n'éprouva plus le besoin de recourir aux services de son mentor, et sans cesser tout à fait, la correspondance se relâcha: aucune lettre entre septembre 1947 et février 1948 et onze lettres seulement, entre mars 1948 et janvier 1951, alors qu'elle s'efforce, mais en vain, d'écrire un troisième roman, dont un seul chapitre parut finalement, sous un titre significatif: «Le plomb dans l'aile» 23 .

21 Voir lettre de Germaine Guèvremont à DesRochers, juillet 1947 [?]: "MarieDidace " est partie depuis vendredi. Non, vous ne rirez pas de moi si je vous parle de mon désemparement, depuis. J'erre, parmi mes papiers, comme une áme en peine. Depuis plus d'une semaine, je me cherchais toutes sortes d'excuses pour retarder la remise du texte à l'imprimeur. Jai da m'y décider.

22 Eugène Issalys, né à Rignac (France), le 16 avril 1884, entre à l'emploi de la Librairie Beauchemin en 1911. Il devient directeur des éditions en 1928; puis administrateur de la maison en 1942. Voir Vedettes (Who's who français), $2^{e}$ éd., Montréal, 1958, p. 142 (3 $3^{\mathrm{e}}$ éd., 1960, p. 147).

23 Germaine Guevremont, "Le plomb dans l'aile", Cahiers de' l'Académie canadienne-française 5: Contes et nouvelles, 1959, p. 69-75. Voir Jean-Pierre Duquette, "Marie-Didace, roman de Germaine Guèvremont (née Grignon)», Dictionnaire des œuvres littéraires du Québec, tome III, 1940-1959, 1982, p. 609-610. 
Germaine Guèvremont avait vécu intensément avec Alphonsine, Angélina, le père Didace et l'Acayenne, mais surtout avec le Survenant, véritable incarnation de ses désirs et de ses phantasmes. Ces personnages une fois disparus, c'est le Chenal du Moine tout entier qui disparaissait à son tour. Quant à Marie-Didace, l'orpheline, elle ne faisait pas le poids. Alors la romancière, incapable de vivre ailleurs que dans le monde qu'elle avait elle-même créé, mais qui avait fini par lui échapper, trouva le moyen de le ressusciter intégralement, grâce à la radio d'abord, puis à la télévision. La formule même du radioroman et du téléroman, avec ses épisodes brefs et circonscrits, convenait on ne peut mieux au talent de conteuse de Germaine Guèvremont. Le public, fidèle jusqu'au bout, lui manifesta son adhésion la plus totale. Mais tout cela est une autre histoire...

La correspondance que nous venons d'examiner nous aura permis de suivre presque pas à pas l'élaboration des divers chapitres de MarieDidace. On a vu avec quelle désinvolture Germaine Guèvremont compose, multipliant les courtes scènes au gré de l'inspiration, bien plus proche en cela du conteur que du romancier, puis liant ces scènes les unes aux autres, mais non sans les avoir parfois aérées, élaguées, modifiées ou transformées, de manière à les articuler fermement, assurant ainsi leur cohérence et leur efficacité dans l'économie générale du récit. Ce travail de patience, ce véritable labeur, ne lui aura cependant pas trop pesé, car elle eut le rare bonheur de connaître un homme qui, avec une abnégation que seules une profonde amité et une absolue confiance peuvent expliquer, accepta de cheminer à ses côtés pendant tout le temps que dura la gestation de l'œuvre. Et cette œuvre, on peut dire qu'elle fut commune: Marie-Didace est le fruit d'une collaboration d'autant plus admirable qu'elle est rare, sous cette forme particulière, dans le monde littéraire. Ce n'est pas le moindre mérite de ces lettres que de nous l'avoir révélé. Et quoique monodique, cette correspondance célèbre la mémoire d'une femme et d'un homme unis par la passion de la littérature. 